\title{
Comparing Two-Dimensional, Axisymmetric, Hybrid-Direct Kinetic and Hybrid Particle-in-Cell Simulations of the Discharge Plasma in a Hall Thruster
}

\author{
Astrid L. Raisanen ${ }^{1}$, Kentaro Hara ${ }^{2}$ and Iain D. Boyd ${ }^{3}$ \\ University of Michigan, Ann Arbor, MI 48109
}

\begin{abstract}
Deterministic, direct kinetic models are of interest in the simulation of plasma physics in a Hall effect thruster because of the lack of statistical noise that inherently exists in particle methods. The present study is concerned with the evaluation of a hybrid-direct kinetic (DK) model through comparison with a hybrid Particle-in-Cell (PIC) model, both of which utilize identical quasi one-dimensional electron fluid algorithms and similar boundary conditions and threshold values for simulation kinetics. The two-dimensional, axisymmetric DK and PIC schemes are employed to model the ions and neutral atoms in a Hall thruster channel and its near-field plume. Results show reasonable agreement in time-averaged plasma properties, suggesting that the hybrid-DK method can be used as an alternative to the standard hybrid-PIC method to model the physics in Hall thrusters. Furthermore, timedependent hybrid-DK properties show smooth behavior that are free of the statistical fluctuations seen in the PIC results. However, analysis of the time-dependent properties shows that the performance of the hybrid-DK method is sensitive to boundary conditions and threshold values. Therefore, further investigation is required to verify that the present hybrid-DK simulation accurately models the evolving physics in a Hall thruster.
\end{abstract}

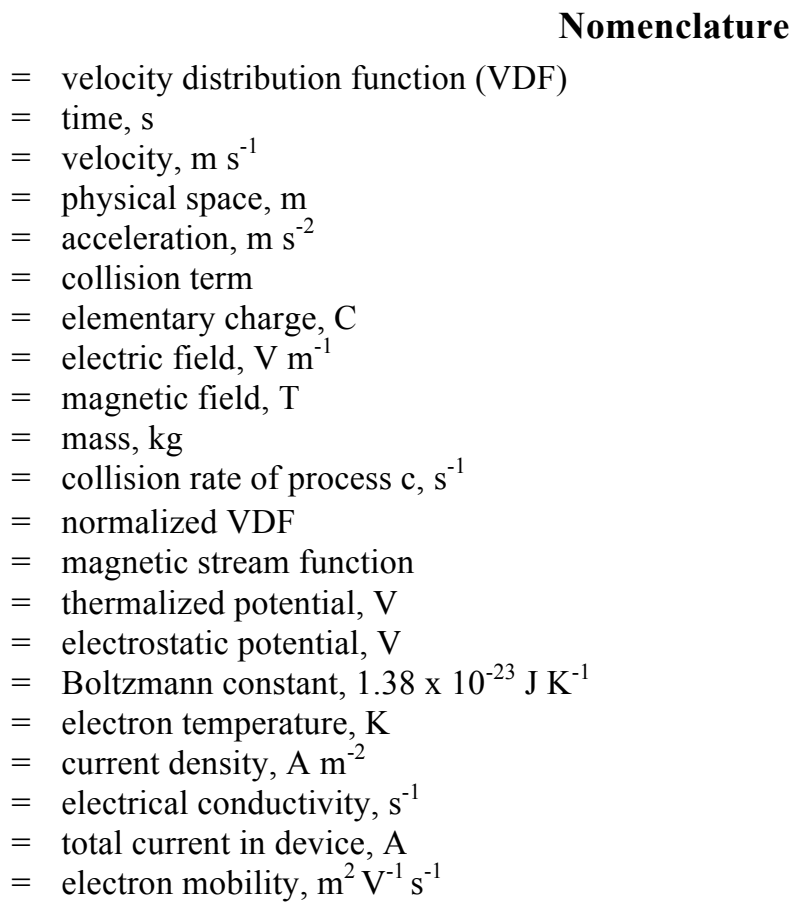

\footnotetext{
${ }^{1}$ Ph.D. Candidate, Department of Aerospace Engineering, Student Member AIAA.

2 Princeton Plasma Physics Laboratory, Princeton, NJ 08543, Member AIAA.

${ }^{3}$ James E. Knott Professor, Department of Aerospace Engineering, Fellow AIAA.

1 of 14
}

American Institute of Aeronautics and Astronautics 


$\begin{array}{lll}n_{e}{ }^{*} & =\text { reference plasma density, } 1 \times 10^{12} \mathrm{~m}^{-3} \\ n_{s} & =\text { number density of species } \mathrm{s}^{-3} \mathrm{~m}^{-3} \\ \mathcal{S} & =\text { lambda-line surface area, } \mathrm{m}^{2} \\ \varepsilon & =\text { mean electron energy, } \mathrm{eV} \\ v(\varepsilon) & =\text { frequency of electron energy losses, } \mathrm{s}^{-1} \\ \forall & =\text { lambda-line volume, } \mathrm{m}^{3} \\ \omega_{B, e} & =\text { electron cyclotron frequency, } \mathrm{s}^{-1} \\ U_{\text {loss }} & =\text { sheath energy, eV } \\ N_{C F L} & =\text { Courant Friedrich Lewy (CFL) number } \\ \dot{m} & =\text { mass flow rate, } \mathrm{kg} \mathrm{s}^{-1} \\ T & =\text { thrust, } \mathrm{N}\end{array}$

\section{Introduction}

$\mathrm{T}$

WO-dimensional, axisymmetric, hybrid-Particle-in-Cell (PIC) algorithms have been utilized to model the discharge plasma in Hall effect thrusters (HETs) since the early 1990s when Fife developed HPHall. ${ }^{1}$ While such simulation methods have been shown to calculate accurate macroscopic thruster properties, including thrust and discharge current, they require various Bohm coefficients in the plasma discharge region to account for anomalous electron transport. Previous studies have quantified the Bohm coefficient for various plasma discharge regions and qualitatively attributed anomalous electron transport to a variety of phenomena including turbulent plasma fluctuations and wall collisionality. ${ }^{2}$ However, to quantify the contribution that electron transport has on dynamically changing, high frequency oscillations seen in Hall thruster operation, PIC-related statistical noise must be reduced. ${ }^{3}$ Since a grid-based kinetic method has no statistical noise, the present study provides a comparison of time-averaged and instantaneous results from a hybrid-DK simulation with results from a hybrid-PIC simulation, thus quantifying the relative effect of probabilistic error on thruster computations.

Section II describes the thruster considered in this work. Sections III and IV discuss the DK and electron algorithms, and Section V discusses the numerical model and its boundary conditions in some detail. Section VI provides simulation results, and Section VII concludes the study, offering insight into future work using the hybridDK simulation methodology.

\section{UM/AFRL P5 Hall Thruster}

The present study considers the $5 \mathrm{~kW}$ class UM/AFRL P5 thruster. Thruster properties used as input to the simulation include geometry, magnetic field configuration, discharge voltage, and input mass flow rate. The experimentally obtained thruster properties alongside published computational results are summarized in Table $1 .^{4}$

Table 1. UM/AFRL P5 Thruster Properties.

\begin{tabular}{|rc|c|c|}
\hline & & Experimental & Koo and Boyd [4]* \\
\hline Mass flow rate & $\dot{m}$ & $10.2 \mathrm{mg} / \mathrm{s}$ & $10.2 \mathrm{mg} / \mathrm{s}$ \\
Discharge Voltage & $\mathrm{V}_{\mathrm{d}}$ & $300 \mathrm{~V}$ & $275 \mathrm{~V}$ \\
Discharge Current & $\mathrm{I}_{\mathrm{d}}$ & $10.0 \mathrm{~A}$ & $11.3 \mathrm{~A}$ \\
Thrust & $\mathrm{T}$ & $180 \mathrm{mN}$ & $179 \mathrm{mN}$ \\
Isp & $I s p$ & $1740 \mathrm{~s}$ & $1790 \mathrm{~s}$ \\
Efficiency & $\eta$ & $51.0 \%$ & $50.5 \%$ \\
\hline
\end{tabular}

To emulate these properties within the simulation, the discharge voltage is set to $V_{d}$ at the anode and $0 \mathrm{~V}$ at the effective cathode. Neutral xenon atoms are injected at the anode location. The experimentally measured magnetic field values are imposed throughout the simulation domain. Since experimental values are not available everywhere, the magnetic field is extrapolated in the upper region of the near-field plume for $0.120 m<r<0.145 \mathrm{~m}$. The hybridDK thruster simulation domain is shown in Fig. 1, and Fig. 2 shows the magnetic field components along the thruster channel centerline. 


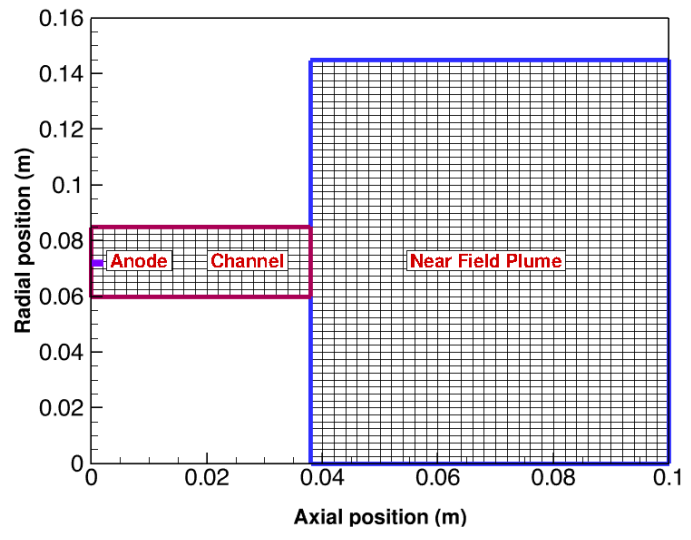

Figure 1. Hybrid-DK thruster simulation domain.

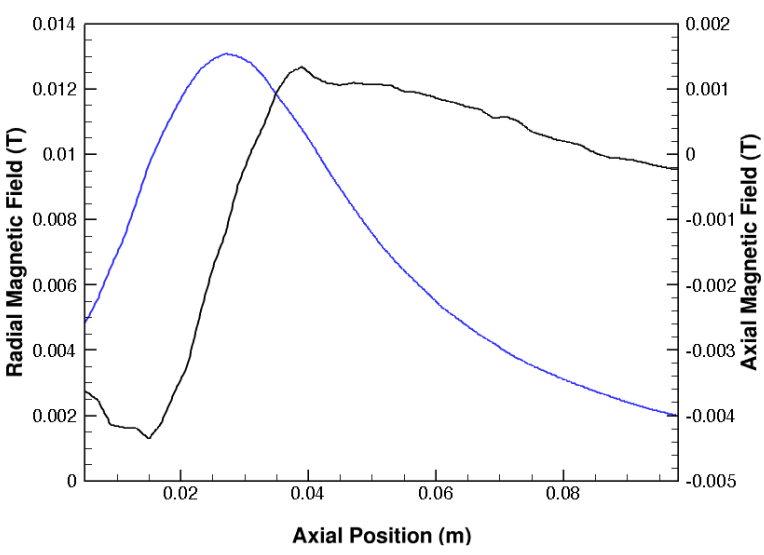

Figure 2. Magnetic field profiles along the thruster channel centerline (blue: radial magnetic field; black: axial magnetic field).

\section{Modeling Ions and Neutrals}

For comparison purposes, this study utilizes the particle-based PIC model that was developed by Koo and Boyd. ${ }^{4}$ In the model, a two-dimensional, divergence-free, static magnetic field is assumed throughout the axial-radial simulation domain. Due to a large Larmor radius compared to channel size, ions are effectively unmagnetized. Thus, ions and neutral atoms are modeled using a PIC technique that simulates the motion of macroparticles via a second order, classical leapfrog update scheme. The depletion of neutral atoms due to ground state ionization is calculated using a Monte Carlo Collision (MCC) model. In the present study, only singly charged ionization is included.

The DK simulation approach employed in this work is a modified, two-dimensional, axisymmetric version of the grid-based kinetic model developed by Hara et al. ${ }^{3}$ This model solves the Boltzmann equation in discretized phase space, evaluating moments of the velocity distribution functions (VDFs) to obtain macroscopic quantities such as ion number density and mean velocity. The Boltzmann equation, in its most general form, is given by:

$$
\frac{\partial f}{\partial t}+\boldsymbol{v} \cdot \frac{\partial f}{\partial z}+\boldsymbol{a} \cdot \frac{\partial f}{\partial v}=S
$$

where $f$ is the VDF, $\boldsymbol{v}$ is the velocity, $\boldsymbol{z}$ is physical space, $t$ is time, $\boldsymbol{a}$ is acceleration and $S$ is the collision term. Note that the collisionless Boltzmann equation is synonymous with the Vlasov equation, which is often used to describe the behavior of high temperature plasmas. The acceleration of a non-relativistic plasma can be written as:

$$
\boldsymbol{a}=e(\boldsymbol{E}+\boldsymbol{v} \times \boldsymbol{B}) / m
$$

where $e$ is the elementary charge, $m$ is the mass, $\boldsymbol{E}$ is the electric field, and $\boldsymbol{B}$ is the magnetic field. In a Hall thruster, $\boldsymbol{B}$ and $\boldsymbol{E}$ are primarily in the axial and radial $(z, r)$ directions. Since the DK simulation is limited to $z$ and $r$, only $\boldsymbol{E}$ contributes to the acceleration term in Eq. (2). The number density and mean velocity are obtained from:

$$
\begin{gathered}
n(\mathbf{z}, t)=\int_{-\infty}^{\infty} f(\mathbf{z}, \boldsymbol{v}, t) d \boldsymbol{v} \\
\boldsymbol{u}(\mathbf{z}, t)=\int_{-\infty}^{\infty} \boldsymbol{v} \cdot \hat{f}(\mathbf{z}, \boldsymbol{v}, t) d \boldsymbol{v}
\end{gathered}
$$

where $\hat{f}=f / n$ is the normalized VDF.

\section{A. Neutral Atom Boltzmann Equation}

Since they carry no charge, neutral atoms do not experience any external force from the electric field. In two dimensions, the neutral Boltzmann equation reduces to:

$$
\frac{\partial f_{n}}{\partial t}+v_{z} \frac{\partial f_{n}}{\partial z}+v_{r} \frac{\partial f_{n}}{\partial r}=S_{n}
$$

3 of 14

American Institute of Aeronautics and Astronautics 


\section{B. Ion Boltzmann Equation}

Ions experience acceleration due to the force from the electric field. As such, the two-dimensional Boltzmann equation for ions is:

$$
\frac{\partial f_{i}}{\partial t}+v_{z} \frac{\partial f_{i}}{\partial z}+v_{r} \frac{\partial f_{i}}{\partial r}+\frac{e E_{z}}{m_{i}} \frac{\partial f_{i}}{\partial z}+\frac{e E_{r}}{m_{i}} \frac{\partial f_{i}}{\partial r}=S_{i}
$$

where the electric field information is obtained from the fluid electron algorithm.

\section{Collisions}

The present model includes singly charged ionization collisions. Assuming that the more massive neutral atoms move much more slowly than electrons, the ionization rate can be approximated as a function of the electron mean energy: ${ }^{5}$

$$
\dot{n}_{\text {ion }}=n_{n} n_{e} \zeta(\varepsilon)
$$

where $n_{n}$ is the neutral number density, and $n_{e}$ is the electron number density. Electron energy-dependent cross sections are obtained from Puech and Mizzi. ${ }^{6}$ For each ionization event, a neutral atom is deleted, and an ion with the identical physical location and velocity is created, conserving the total number of particles in the system. The collision terms for ions and neutral atoms are described by $S_{i}$ and $S_{n}$, respectively.

$$
\begin{gathered}
S_{i}(\boldsymbol{v}, \mathbf{z}, t)=\dot{n}_{i o n} \hat{f}_{n}(\boldsymbol{v}) \\
S_{n}(\boldsymbol{v}, \mathbf{z}, t)=-\dot{n}_{\text {ion }} \hat{f}_{n}(\boldsymbol{v})
\end{gathered}
$$

\section{Quasi 1D Electron Fluid Model}

The DK model for neutral atoms and ions requires the electrostatic potential, plasma number density, and electron mean energy as inputs for the algorithm at each global time step in the Cartesian domain. These quantities are calculated via a quasi one-dimensional electron fluid model, which is solved on a smaller timescale than the DK model. The electron model for this study is extracted from Koo and Boyd's hybrid-PIC model ${ }^{4,7}$ and tested to ensure consistency.

\section{A. Electrostatic Potential}

Since the electron diffusion coefficient is much higher along magnetic field lines than across them, a balance of pressure and electric forces is considered only along magnetic field lines. Furthermore, electron dynamics in the discharge region are much more rapid than the dynamics of more massive particles, allowing for the approximation of electron thermal equilibrium along magnetic field lines. These two properties permit the applicability of the thermalized potential throughout the domain:

$$
\phi^{*}(\lambda)=\phi-\frac{k_{B} T_{e}}{e} \ln \left(n_{e}\right)
$$

where $\phi^{*}(\lambda)$ is the thermalized potential of the magnetic stream function, $\phi$ is the electrostatic potential, $k_{B}$ is Boltzmann's constant, and $T_{e}$ is the electron temperature in Kelvin. By discretizing the domain using magnetic field lines, it is possible to solve the one-dimensional Ohm's Law $(J=\sigma E)$ from the virtual anode line, $\lambda_{a}$, to the virtual cathode line, $\lambda_{c}$. Current conservation dictates that $\phi^{*}$ adheres to the following equation:

$$
\begin{aligned}
\phi^{*}\left(\lambda_{c}\right)-\phi^{*}\left(\lambda_{a}\right)=-I_{T} \sum_{\lambda=\lambda_{a}}^{\lambda=\lambda_{c}} \frac{1}{\int_{\mathcal{S}} e n_{e} \mu r B \partial \mathcal{S}} d \lambda-\sum_{\lambda=\lambda_{a}}^{\lambda=\lambda_{c}} \frac{\int_{\mathcal{S}} e n_{e} \mu r B\left[\ln \left(\frac{n_{e}}{n_{e}{ }^{*}}-1\right)\right] \frac{k_{B}}{e} \frac{\partial T_{e}}{\partial \lambda} \partial \mathcal{S}}{\int_{\mathcal{S}} e n_{e} \mu r B \partial \mathcal{S}} d \lambda \\
+\sum_{\lambda=\lambda_{a}}^{\lambda=\lambda_{c}} \frac{\int_{\mathcal{S}} e n_{i} u_{i} \partial \mathcal{S}}{\int_{\mathcal{S}} e n_{e} \mu r B \partial \mathcal{S}} d \lambda
\end{aligned}
$$

where $I_{T}$ is the total current in the device; $\mu$ is the electron mobility; $n_{e}{ }^{*}$ is a reference plasma density, typically chosen to be $1 \times 10^{12} \mathrm{~m}^{-3}$; and $\mathcal{S}$ represents the area about a given field line. Equation (11) represents a closed form

4 of 14

American Institute of Aeronautics and Astronautics 
solution for the total current, from which the thermalized potential gradient is retrieved. $\phi$ is calculated using Eq. (10), and the electric field, $\boldsymbol{E}$, is then evaluated via:

$$
\boldsymbol{E}=-\nabla \phi
$$

\section{B. Electron Energy}

The electron energy algorithm in Koo's work is nearly identical to the method used by Fife in HPHall. ${ }^{7}$ Taking into consideration advection, diffusion, ohmic heating, and energy loss, the volume-integrated electron energy equation over all $\lambda$ is:

$$
\begin{aligned}
\iiint \frac{\partial}{\partial t}\left(n_{e} \varepsilon\right) d \forall+\iiint & \nabla \cdot\left(\frac{5}{3} n_{e} \varepsilon \boldsymbol{v}_{e}\right) d \forall-\iiint \nabla \cdot\left(\frac{10 n_{e} \mu_{e, \perp} \varepsilon}{9} \nabla \varepsilon\right) d \forall \\
= & -\iiint n_{e} \boldsymbol{v}_{e} \cdot \boldsymbol{E} d \forall-\iiint n_{e} \varepsilon v(\varepsilon) d \forall
\end{aligned}
$$

where $\varepsilon$ is the electron mean energy in $\mathrm{eV}\left(e \varepsilon=\frac{3}{2} k_{B} T_{e}\right), \mu_{e, \perp}$ is the electron mobility perpendicular to magnetic field lines, and $v(\varepsilon)$ represents the frequency of electron energy losses associated with ionization and wall collisions. In the present study, the electron-wall energy loss is based on a model used by Boeuf and Garrigues: ${ }^{8}$

$$
v(\varepsilon)_{\text {walls }}=\alpha \cdot 10^{7} \exp \left(\frac{-U_{\text {loss }}}{\varepsilon}\right)
$$

where $\alpha=0.20$ corresponds to the frequency of electron collisions with the wall sheath, and $U_{\text {loss }}=20 \mathrm{eV}$ represents the sheath energy. Equation (13) is derived under the assumption that the electron energy distribution function (EEDF) is nearly Maxwellian, therefore justifying the use of a single fluid approximation in which the EEDF is described by the mean electron energy.

\section{Electron Mobility}

Electron mobility perpendicular to magnetic field lines has the classical description:

$$
\mu_{e, \perp}=\frac{e}{m_{e} v_{m}} \frac{1}{1+\frac{\omega_{B, e}^{2}}{v_{m}^{2}}}
$$

where $\omega_{B, e}$ is the electron cyclotron frequency, and $v_{m}$ is the total electron momentum transfer frequency. In this case, the electron-neutral collision frequency is evaluated as: ${ }^{4}$

$$
v_{m}=n_{n} \cdot 2.5 \times 10^{-13}
$$

However, the classical electron mobility alone is not sufficient to explain experimental results. In this study, "anomalous electron transport" is implemented via a thruster-specific, semi-empirical correction to the collision frequency. The details regarding the semi-empirical correction can be found in Koo and Boyd's work; resultant bestfit thruster mobility values utilized in the present work are shown in Fig. 3. ${ }^{4}$

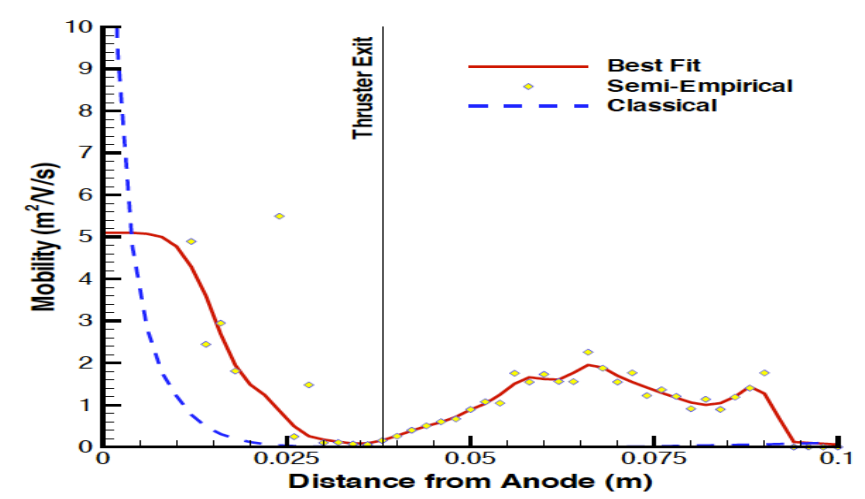

Figure 3. Best-fit mobility values for UM/AFRL P5 thruster. ${ }^{4}$

5 of 14

American Institute of Aeronautics and Astronautics 


\section{Two-dimensional Axisymmetric Hybrid-DK Simulation}

This study employs a two-dimensional Vlasov solver coupled with a quasi one-dimensional fluid electron solver to model the plasma discharge in a Hall thruster channel and near-field plume. The hybrid-DK solver is subjected to boundary conditions similar to those used in Koo's hybrid-PIC simulation in order to properly compare the results.

\section{A. Direct Kinetic (Vlasov) Solver}

As previously mentioned, the DK solver methodology is an adaptation of that used in Hara's previous work. ${ }^{3}$ There are four dimensions in the DK solver, i.e. two dimensions in physical space and two dimensions in velocity space. As such, the physical and velocity updates are split, similar to a leapfrog scheme often used in particle methods. The time integration is a second-order accurate Runge Kutta (RK2) method without any dimensional splitting. The collisionless Boltzmann, or Vlasov, equation can be written as:

$$
\frac{\partial f}{\partial t}+L\left(f\left(z, r, v_{z}, v_{r}\right)\right)=0
$$

where $L$ consists of the advection terms in Eq. (5) and Eq. (6). A second-order Runge Kutta method is described by:

$$
\begin{gathered}
f^{*}=f^{n}+\Delta t L\left(f^{n}\right) \\
f^{n+1}=f^{n}+\frac{\Delta t}{2}\left[L\left(f^{n}\right)+L\left(f^{*}\right)\right]
\end{gathered}
$$

In this simulation, $i z$ and $i r$ represent cell numbers in $z$ and $r ; j z$ and $j r$ represent cell numbers in $v_{z}$ and $v_{r}$, respectively. The discretized flux terms are written as:

$$
\begin{aligned}
v_{z} \frac{\partial f}{\partial z} & =\frac{v_{z}}{\Delta z}\left(f_{i z+1 / 2, i r, j z, j r}-f_{i z-1 / 2, i r, j z, j r}\right) \\
v_{r} \frac{\partial f}{\partial r} & =\frac{v_{r}}{\Delta r}\left(f_{i z, i r+1 / 2, j z, j r}-f_{i z, i r-1 / 2, j z, j r}\right) \\
a_{z} \frac{\partial f}{\partial v_{z}} & =\frac{a_{z}}{\Delta v_{z}}\left(f_{i z, i r, j z+1 / 2, j r}-f_{i z, i r, j z-1 / 2, j r}\right) \\
a_{r} \frac{\partial f}{\partial v_{r}} & =\frac{a_{r}}{\Delta v_{r}}\left(f_{i z, i r, j z, j r+1 / 2}-f_{i z, i r, j z, j r-1 / 2}\right)
\end{aligned}
$$

where the subscripts $+1 / 2$ and $-1 / 2$ denote the cell interfaces. To calculate the flux, a finite volume method with a modified Arora-Roe limiter is used. ${ }^{3}$ For numerical stability, the time step is restricted by the Courant FriedrichLewy (CFL) condition and, for the RK2 method, must satisfy:

$$
N_{C F L}=\max \left(\frac{v_{Z} \Delta t}{\Delta z}+\frac{v_{r} \Delta t}{\Delta r}+\frac{a_{Z} \Delta t}{\Delta v_{z}}+\frac{a_{r} \Delta t}{\Delta v_{r}}\right) \leq \beta
$$

If the left hand side of Eq. (24) is greater than $\beta$, the time step is reduced as $\Delta t=\Delta t / N_{C F L}$ until the CFL condition is satisfied. $\beta$ is generally set near the value of one.

The DK simulation is parallelized for an even number of processors using message passing interface (MPI). The computational domain is divided evenly in space between the channel, the near field plume directly adjacent to the channel, the lower field plume, and the upper field plume. Information is exchanged via buffer cells located at processor boundaries. No domain decomposition is applied in velocity space because the moments of the VDFs, such as the number density and mean velocity, need to be calculated for the collision rate and electron fluid model.

\section{B. Quasi One-Dimensional Electron Solver}

The quasi one-dimensional electron solver is not the focus of the current work. The solver is identical to that used in Koo's previous work and utilizes the magnetic field stream function with equally spaced $\lambda$-intervals to enable discretization of the two-dimensional, axisymmetric domain into a one-dimensional domain. ${ }^{4}$ The location between the anode and cathode lines is the active domain, i.e. the region over which the electron energy equation is solved. $\lambda_{a}$ should be located upstream of the ionization zone, and $\lambda_{c}$ should be placed near the effective cathode

6 of 14

American Institute of Aeronautics and Astronautics 
plane, which is typically located at a local potential minima, well downstream of the acceleration zone. In this case, the anode line intersects the $\mathrm{z}$-axis at $\mathrm{z}=0.005 \mathrm{~m}$, and the cathode line intersects the $\mathrm{z}$-axis very near the end of the domain.

\section{Domain Size and Discretization}

The simulation input values and grid discretization are shown in Table 2. Note that the electron algorithm is solved in a rectangular domain of size $\left[L_{z}, L_{r}\right]$ while the DK algorithm's simulation domain includes the thruster channel and near-field plume shown in Fig. 1. However, the cell sizes and locations between the shared grids are identical so that cell-averaged information can be directly exchanged at each global time step. The DK algorithm for the present case is solved using 24 processors. In the electron model, the cell-centered average velocity, ion density, and neutral density inputs from the DK algorithm are interpolated to nodal locations using standard shape factors specified by Ruyten. ${ }^{9}$ The global time step for the DK solver is set to $1.0 \times 10^{-8}$ seconds. For consistency, the hybridPIC simulation has an identical global time step, and both simulations utilize an electron time step of $5.0 \times 10^{-11}$ seconds.

Table 2. Hybrid-DK Grid Discretization.

\begin{tabular}{|rrc|}
\hline Channel Length & $\mathrm{L}_{\mathrm{ch}}$ & $38 \mathrm{~mm}$ \\
Channel Diameter & $\mathrm{D}_{\mathrm{ch}}$ & $25 \mathrm{~mm}$ \\
Axial Domain Length & $\mathrm{L}_{\mathrm{z}}$ & $100 \mathrm{~mm}$ \\
Radial (Plume) Domain Length & $\mathrm{L}_{\mathrm{r}}$ & $145 \mathrm{~mm}$ \\
Neutral atom velocity space & {$\left[\mathrm{v}_{\mathrm{z}, \min }, \mathrm{v}_{\mathrm{z}, \max }\right],\left[\mathrm{v}_{\mathrm{r}, \mathrm{min}}, \mathrm{v}_{\mathrm{r}, \mathrm{max}}\right]$} & {$[-1200 \mathrm{~m} / \mathrm{s}, 1500 \mathrm{~m} / \mathrm{s}],[-1200 \mathrm{~m} / \mathrm{s}, 1200 \mathrm{~m} / \mathrm{s}]$} \\
Ion velocity space & {$\left[\mathrm{v}_{\mathrm{z}, \min }, \mathrm{v}_{\mathrm{z}, \max }\right],\left[\mathrm{v}_{\mathrm{r}, \mathrm{min}}, \mathrm{v}_{\mathrm{r}, \mathrm{max}}\right]$} & {$[-7500 \mathrm{~m} / \mathrm{s}, 30000 \mathrm{~m} / \mathrm{s}],[-7500 \mathrm{~m} / \mathrm{s}, 7500 \mathrm{~m} / \mathrm{s}]$} \\
Cell size in Physical space & {$[\Delta \mathrm{z}, \Delta \mathrm{r}]$} & {$[2.0 \mathrm{~mm}, 2.5 \mathrm{~mm}]$} \\
Bins in physical space & $\mathrm{N}_{\mathrm{z}, \mathrm{r}}$ & 1988 \\
Bins in atom velocity space & $\mathrm{N}\left(\mathrm{v}_{\mathrm{z}}\right), \mathrm{N}\left(\mathrm{v}_{\mathrm{r}}\right)$ & 108,240 \\
Bins in ion velocity space & $\mathrm{N}\left(\mathrm{v}_{\mathrm{z}}\right), \mathrm{N}\left(\mathrm{v}_{\mathrm{r}}\right)$ & 250,150 \\
Number of $\lambda-$-lines & $\mathrm{N}_{\lambda}$ & 24 \\
\hline
\end{tabular}

\section{Boundary Conditions}

In both simulations, the incoming neutral atoms are emitted from an annular opening with a height of $0.2 \mathrm{~mm}$ located at the anode side of the thruster, centered at $[\mathrm{r}=0.0725 \mathrm{~m}]$. The velocity of the injected particles is described by a biased Maxwellian velocity distribution with an assumed anode reservoir temperature of $700 \mathrm{~K}$. For the DK simulation, specular and diffuse reflections of neutral particles are considered at the walls of the thruster, and particles leave the domain when they exit the plume region. The accommodation coefficient is 0.90 . Presently, the hybrid-PIC simulation is fully diffuse. In either case, ions recombine to atoms when they hit the thruster walls or pole pieces. The channel chamber temperature is assumed to be $700 \mathrm{~K}$. Although the electron equations are solved only between the anode $\lambda$-line and cathode $\lambda$-line, ionization is allowed to occur throughout the domain. Note that the electron energy outside of the active region is set to $1.0 \mathrm{eV}$. Based on Koo and Boyd's setup configuration of the hybrid-PIC domain, the potential is set to the maximum value of $275 \mathrm{~V}$ at the left side of the domain, and a Dirichlet boundary condition $[\phi=0 \mathrm{~V}]$ is also applied at the right hand side of the domain. ${ }^{4}$ The total potential drop of $275 \mathrm{~V}$ accounts for an assumed cathode fall of $25 \mathrm{~V}$ in the operation of the real thruster. Note that the voltage is allowed to drop slightly below $0 \mathrm{~V}$ within the domain, if necessary. If this is the case, the voltage will achieve a minimum value at the cathode line and then increase in order to reach $0 \mathrm{~V}$ at the end of the computational domain.

Initially, a uniform neutral atom density of $n_{n}=1.0 \times 10^{20} \mathrm{~m}^{-3}$ is applied inside the channel, and $n_{n}=1.0 \times 10^{18}$ $\mathrm{m}^{-3}$ is applied in the plume. This study also applies a uniform ion density of $n_{i}=5.0 \times 10^{15} \mathrm{~m}^{-3}$ inside the channel and $n_{i}=1.0 \times 10^{15} \mathrm{~m}^{-3}$ in the plume. Furthermore, to maintain similarity, both simulations are subjected to the following minimum threshold values: $n_{i, \text { minimum }}=1.0 \times 10^{15} \mathrm{~m}^{-3}$ and $n_{n, \text { minimum }}=1.0 \times 10^{16} \mathrm{~m}^{-3}$, which are important for particle methods as there may be cells that do not contain any macroparticles. Note that the discontinuous initial density distribution is not ideal since it takes approximately $0.3 \mathrm{~ms}$ of simulation time for the discontinuous behavior to become smooth. In the future, the DK simulation will first run for a short period of time without any plasma generation so that neutral particles enter from the anode to fill the domain, similar to the initialization method in HPHall. ${ }^{10}$

In the hybrid-PIC simulation, thermal velocities for both neutral atoms and ions are assigned initially by random sampling from a Maxwellian VDF corresponding to a background neutral temperature of $1000 \mathrm{~K}$. Velocities in the hybrid-DK domain are initially given a normalized Maxwellian distribution in each cell. The hybrid-PIC simulation

7 of 14

American Institute of Aeronautics and Astronautics 
maintains approximately 100,000 ion macroparticles (40+ ion macroparticles/cell on average) and 250,000 neutral macroparticles in the domain, injecting neutral macroparticles at each time step to simulate a constant mass flow rate from the anode.

\section{Results}

The two-dimensional hybrid-DK test case is simulated for $1.0 \mathrm{~ms}$ of physical time, and time-averaged results are compared to those from the hybrid-PIC simulation. The present study also analyzes instantaneous results for the two separate cases. The hybrid-DK simulation requires a wall time of approximately seven days using 24 processors. Since the electron algorithm is combined with the DK algorithm, a check is performed to ensure that the electron algorithm does not make up a significant portion of the computational load. The electron algorithm takes less than $0.05 \%$ of the computational time, indicating that the DK algorithm consumes most of the computational resources, as expected. The serial hybrid-PIC simulation requires a wall time of approximately two days. The computational cost for the DK simulation is much higher than the cost for the PIC simulation due to the discretization of phase space. Since the cost for the model scales with $\mathrm{N}_{\mathrm{z}, \mathrm{r}} \mathrm{N}_{\mathrm{v}}$, a serial DK model would be expected to take approximately 350 times longer than the PIC simulation. This estimate assumes that the PIC simulation maintains approximately 180 macroparticles per cell on average, and the DK simulation maintains $108 \times 240$ neutral velocity bins and $250 \times$ 150 ion velocity bins in each physical cell. Although the DK simulation is not perfectly efficient, parallelization makes the computational time much more manageable.

\section{A. Time-Averaged Plasma Properties}

Time-averaged results are evaluated at $t=1.0 \mathrm{~ms}$ with a sampling interval of $1.0 \times 10^{-3} \mathrm{~ms}$ and are shown in Figs. 4-5. As shown in Fig. 4, in the diffusion region of the channel, which lies in the vicinity of the anode, there are many neutral atoms, relatively few ions, and very little ionization. This is because the voltage is nearly constant, and there is negligible electron energy. Slightly downstream of the anode region, ionization occurs due to the increased electron energy. Since the electric field is still small, ions build up in the ionization region. Shortly thereafter, the particles are accelerated by the large electric field, which corresponds to a steep drop in potential. Since the electron mobility is very low due to the strong magnetic field at the channel exit, the potential falls off quickly to maintain a constant current. Trapped electrons are heated and contribute to the high electron mean energy slightly upstream of the channel exit plane.

While the two sets of solutions show similar qualitative trends, the averaged hybrid-DK results are generally higher than the hybrid-PIC results. The hybrid-DK average electron energy varies more significantly, particularly just upstream of the channel exit, and the average ion density is greater in both the ionization region and the near field plume. These differences are discussed in more detail in subsections B and $\mathrm{C}$.

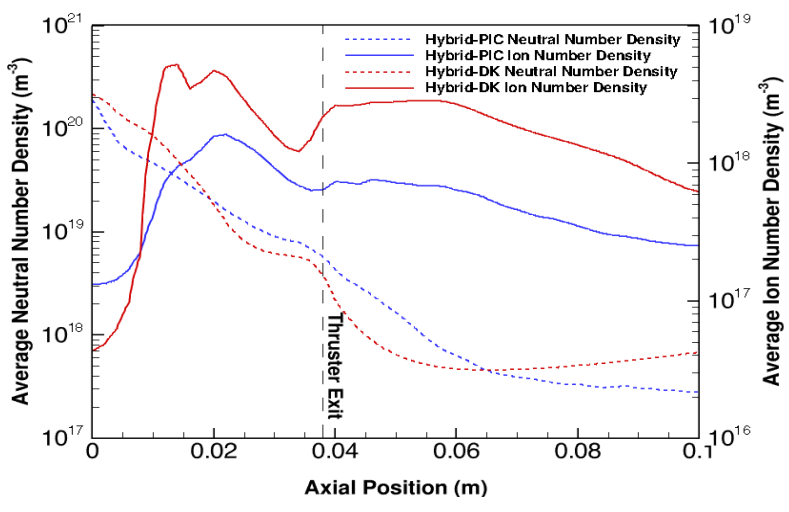

Figure 4. Axial profiles of time-averaged number density along the channel centerline.

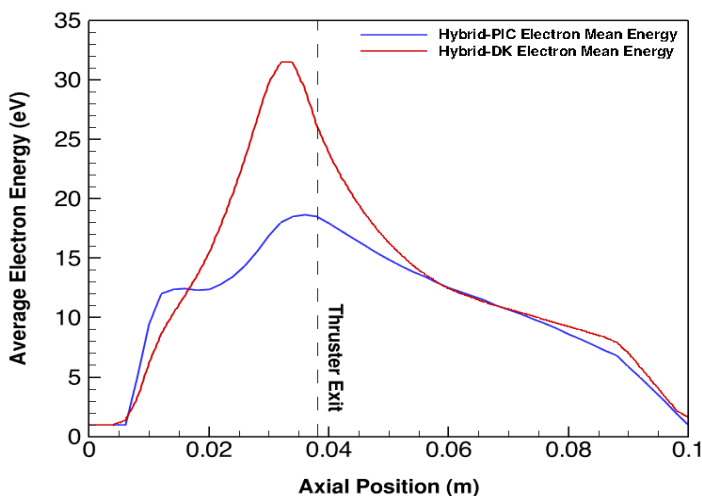

Figure 5. Axial profiles of time-averaged electron energy along the channel centerline.

\section{B. Hybrid-DK Instantaneous Plasma Properties}

Instantaneous plasma properties for the hybrid-DK case are shown at $\mathrm{t}=1.0 \times 10^{-6} \mathrm{~s}$ and $\mathrm{t}=5.0 \mathrm{x} 10^{-5} \mathrm{~s}$. It is clear that the initial number density profile is set up properly, although the discontinuous distribution of particles affects the evolution of the DK results in time. This is evident in the ion number density distribution shown in Fig. 6. Since the density must evolve continuously in space, it first increases both upstream and downstream of the channel

8 of 14

American Institute of Aeronautics and Astronautics 
exit. However, by $0.05 \mathrm{~ms}$ of physical time, the DK simulation exhibits a clear peak in ion number density in the ionization region of the discharge channel, as shown in Fig. 9. Outside of the channel, the ion density again increases, but this is in part due to the fact that the neutral number density achieves its minimum threshold value just upstream of the channel exit and is then artificially maintained at $10^{16} \mathrm{~m}^{-3}$, inflating the ionization rate in the plume in Fig. 11, particularly when compared to the ionization rate in Fig. 8, and producing more ions. Recall that the minimum threshold for the ion and neutral number density was originally set in the hybrid-PIC simulation and was therefore also installed in the hybrid-DK simulation to maintain consistency. The corresponding electron energy distribution in Fig. 10 exhibits a clear maximum just upstream of the channel exit, but the gradient in electron energy is much larger than that exhibited earlier in Fig. 7.

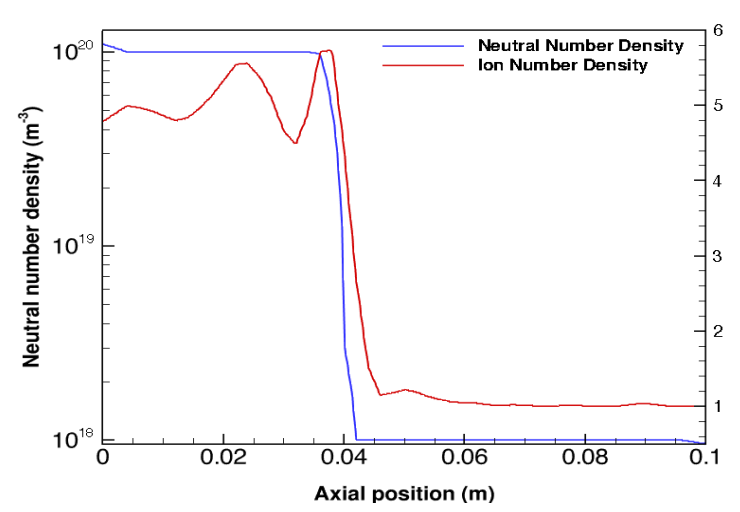

Figure 6. Instantaneous profiles along the channel centerline of number density from the $D K$ simulation at $t=$ $1.0 \times 10^{-6} \mathrm{~s}$.

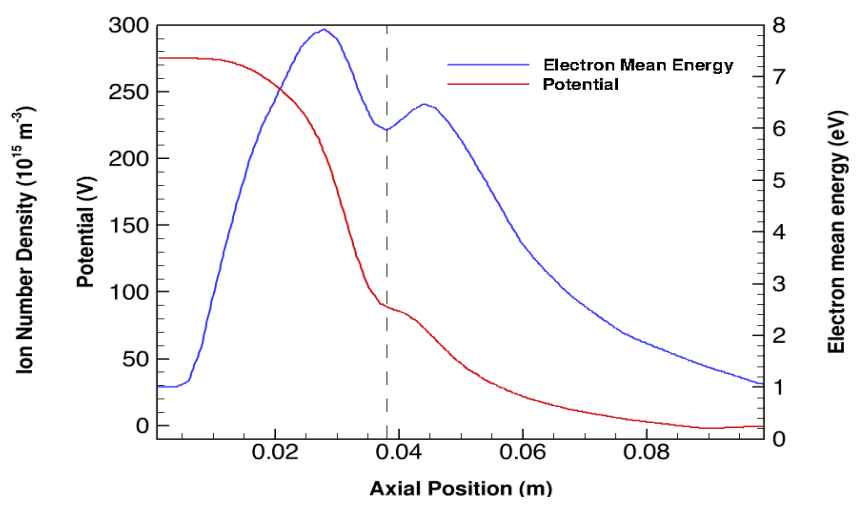

Figure 7. Instantaneous profiles along the channel centerline of electron mean energy and potential from the DK simulation at $\mathrm{t}=1.0 \times 10^{-6} \mathrm{~s}$.

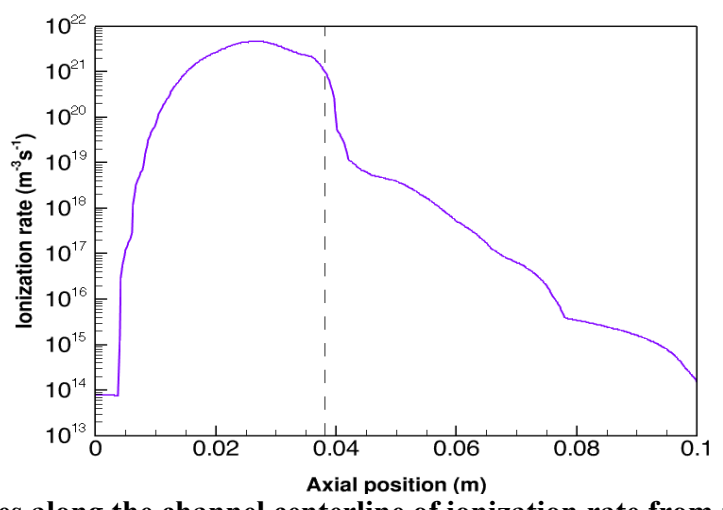

Figure 8. Instantaneous profiles along the channel centerline of ionization rate from the DK simulation at $t=1.0 \times 10^{-6} \mathrm{~s}$. 


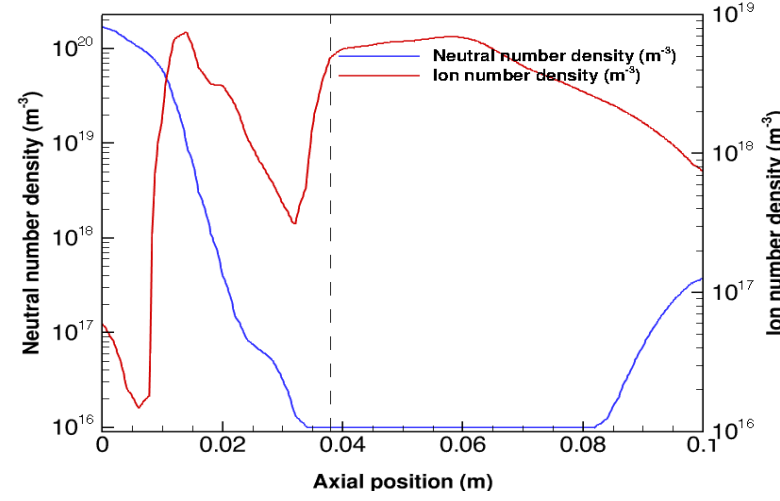

Figure 9. Instantaneous profiles along the channel centerline of number density from the DK simulation at $t=$ $5.0 \times 10^{-5} \mathrm{~s}$.

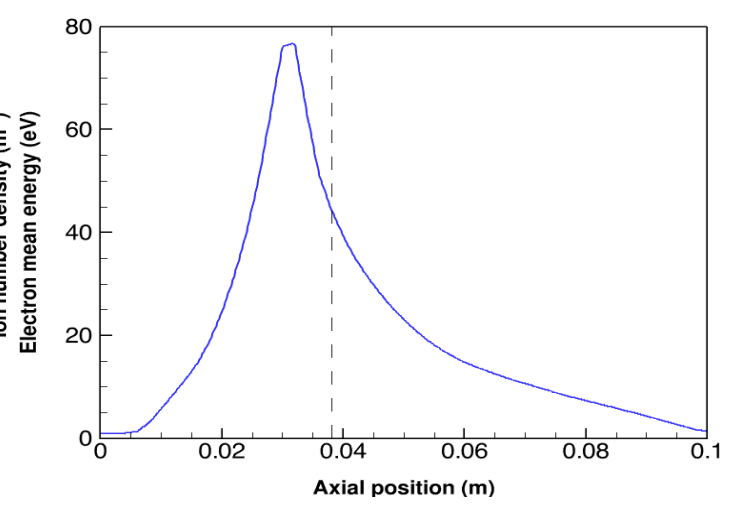

Figure 10. Instantaneous profiles along the channel centerline of the electron mean energy from the DK simulation at $\mathrm{t}=\mathbf{5 . 0} \times 10^{-5} \mathrm{~s}$.

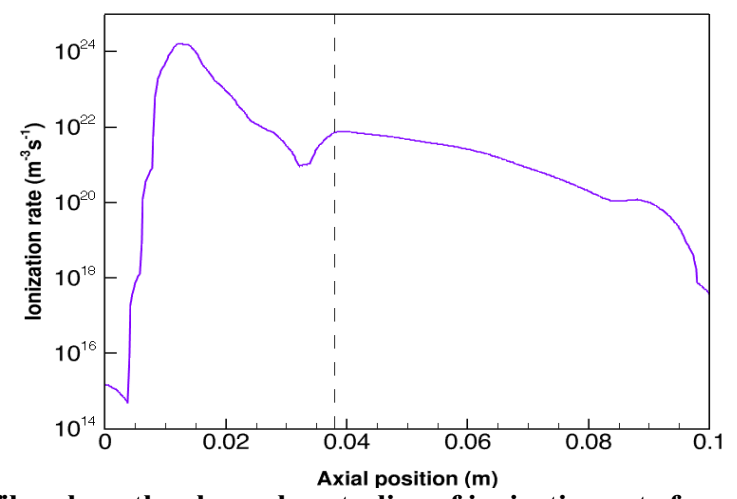

Figure 11. Instantaneous profiles along the channel centerline of ionization rate from the DK simulation at $t=5.0 \times 10^{-5} \mathrm{~s}$.

While it is apparent that the present simulation shows some nonphysical behavior likely due to the imposed simulation threshold values, results in Fig. 12 show that the discharge current is coherent and repeating. In order to more clearly observe the plasma physics in the thruster, the evolution of the ion number density and axial velocity are plotted at different values for the discharge current, which are shown in Fig. 13. Due to availability of data, results are shown for different oscillations, but since the evolution of the discharge current is similar for each oscillation, plasma properties should be representative of behavior over a distinct oscillation. At point 1 in Fig. 13, new plasma is being created, which corresponds to an increase in density between Figs. 14 and 15. At points 5, 4, and 3, the ion density is high, as reflected in Figs. 15-17, and the bulk plasma begins to leave the channel. Properties at point 2, shown in Fig. 18, are similar to those at point 1.

The discharge current is consistently higher than the nominal expected value of $10 \mathrm{~A}$, and the changes in both ion density and ion axial velocity in Figs. 14-18 also appear to be restricted. Results show a minimum threshold for the discharge current around $10 \mathrm{~A}$, which corresponds to the properties at point 4 in Fig. 16. This minimum discharge current is likely nonphysical. It is possible that the imposed minimum neutral number density restricts the ion number density too much in the channel, as shown in Fig. 9. The limited range of the ion exit velocities in Figs. 14-18 supports this constraint on ion density. In order to conserve mass, the channel exit velocity cannot change much if the ion density does not change. The discharge current is directly proportional to the ion number density, per Eq. (11), and if the ion density is restricted within the channel, the minimum discharge current will also be limited, and the physics of the plasma in the channel cannot evolve correctly. 


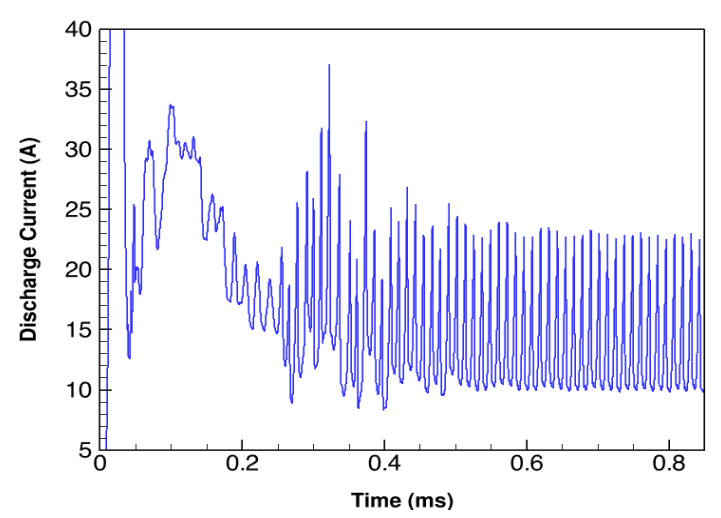

Figure 12. Hybrid-DK discharge current vs. time.

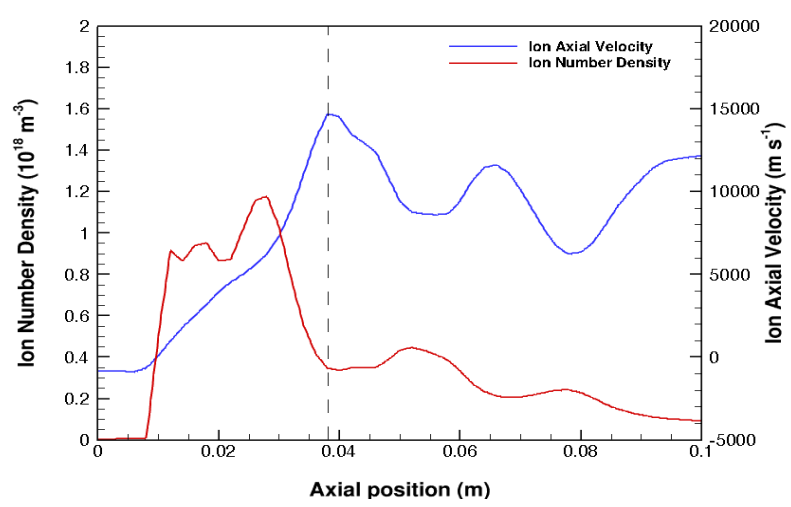

Figure 14. Profiles along the channel centerline of ion number density and ion mean velocity for the DK simulation at point $1(t=0.56 \mathrm{~ms})$.

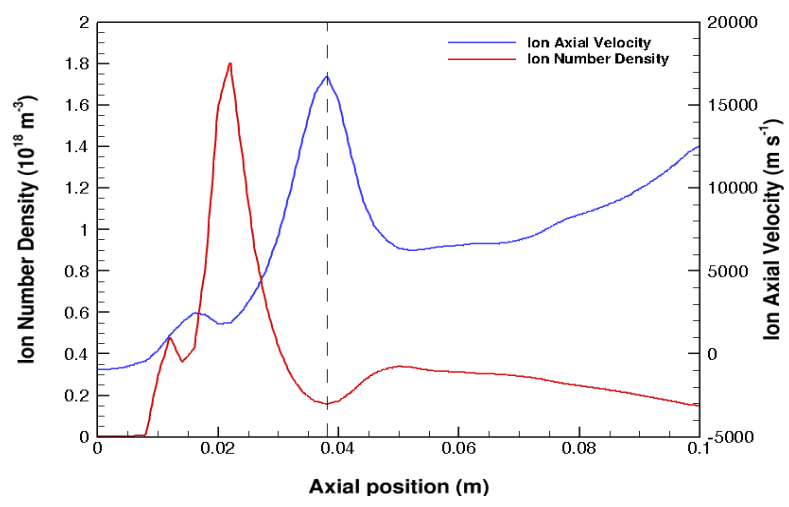

Figure 16. Profiles along the channel centerline of ion number density and ion mean velocity for the DK simulation at point $4(t=0.59 \mathrm{~ms})$.

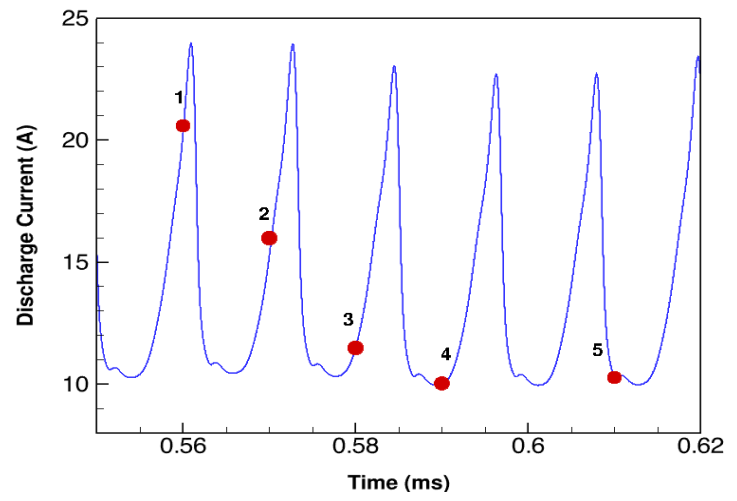

Figure 13. Hybrid-DK discharge current vs. time.

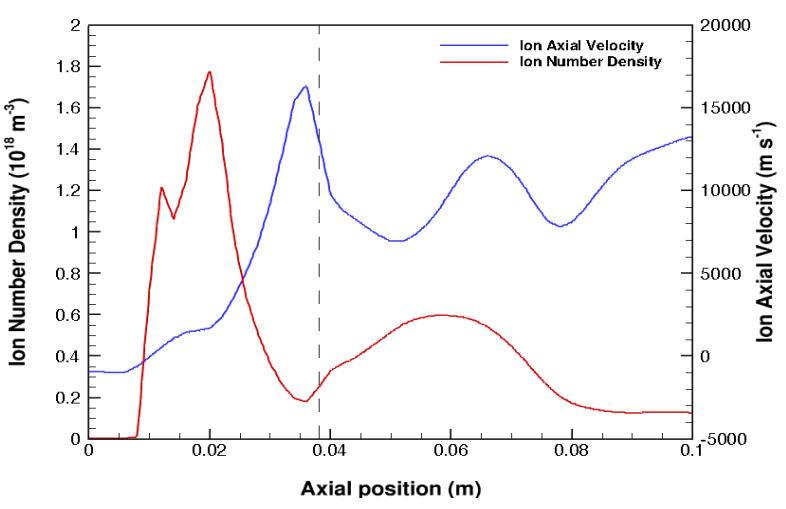

Figure 15. Profiles along the channel centerline of ion number density and ion mean velocity for the DK simulation at point $5(t=0.61 \mathrm{~ms})$.

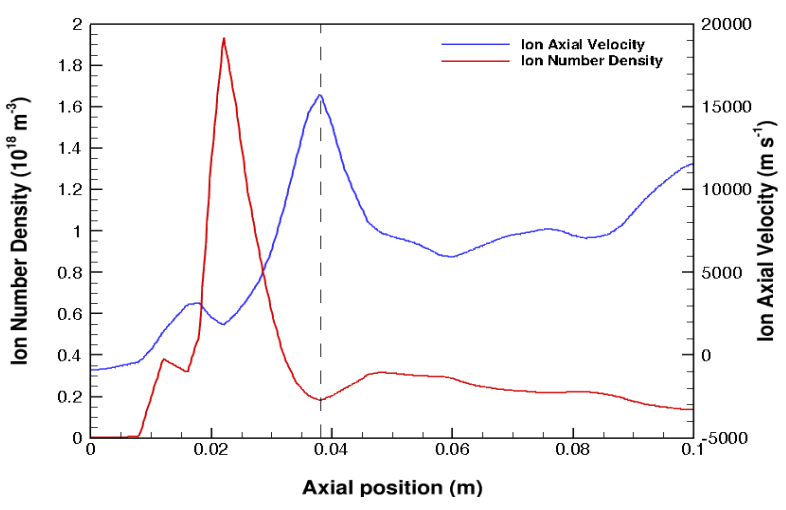

Figure 17. Profiles along the channel centerline of ion number density and ion mean velocity for the DK simulation at point $3(t=0.58 \mathrm{~ms})$. 


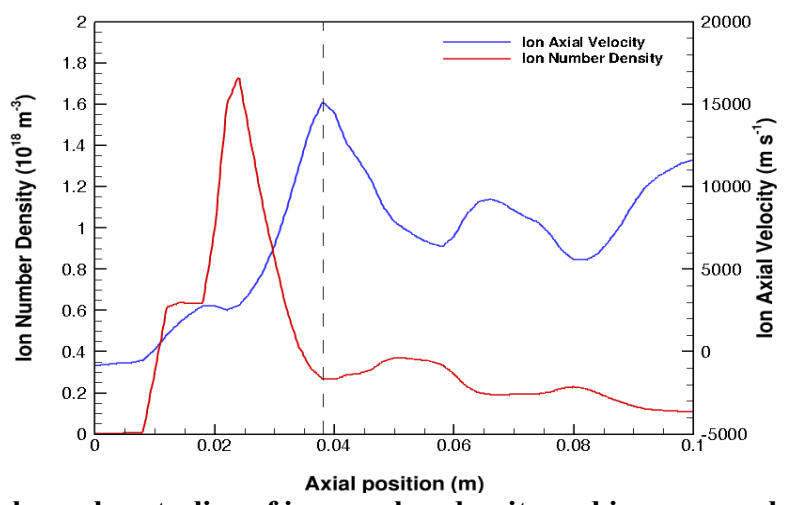

Figure 18. Profiles along the channel centerline of ion number density and ion mean velocity for the DK simulation at point $2(t=0.57 \mathrm{~ms})$.

\section{Hybrid-PIC Instantaneous Plasma Properties}

The evolution of the hybrid-PIC discharge current over time is shown in Fig. 19. While the current does not evolve as coherently as that in the hybrid-DK case, it does have structure. Koo and Boyd's results indicate that there should be more structure in the hybrid-PIC case, and the mean current should be higher. ${ }^{4}$ However, these simulation results do not include doubly charged collisions, and this study confirmed that the difference in discharge current due to the lack of doubly charged collisions is significant. At locations in time shown in Fig. 20, instantaneous plasma properties are evaluated.

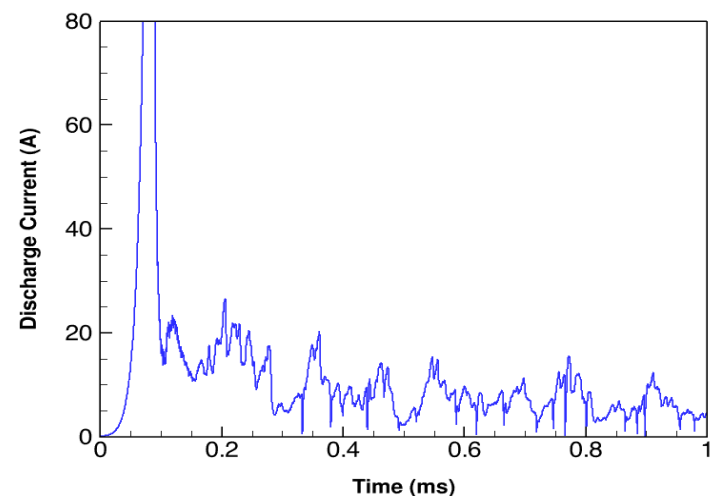

Figure 19. Hybrid-PIC discharge current vs. time.

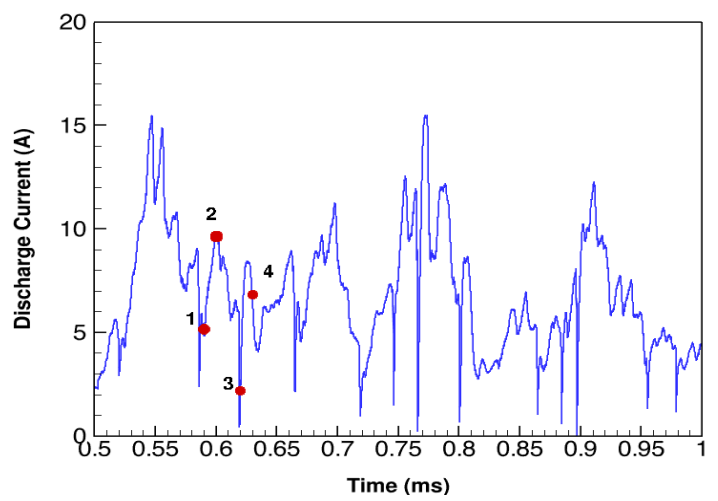

Figure 20. Hybrid-PIC discharge current vs. time.

At point 1 in Fig. 20, bulk plasma has already been created in the channel and is beginning to accelerate across the exit plane, as shown in Fig. 21. At point 2, the ion density is still high, and the exit velocity continues to increase according to Fig. 22. From point 3 to 4, the ion density decreases significantly as bulk plasma leaves the channel, as shown in Figs. 23-24.

While the results make sense, the average hybrid-PIC discharge current is lower than that measured for the DK case, and the reason for this is not entirely clear. It is possible that the installed minimum threshold values affect the DK case significantly but do not have any affect on the PIC results. Therefore, additional investigation with slightly different boundary conditions and threshold values is required, and the contribution of the electron and ion currents should also be quantified. 


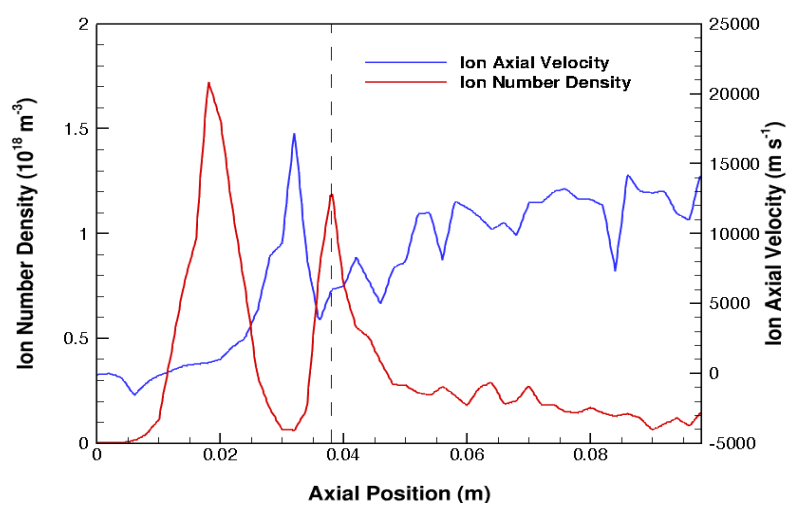

Figure 21. Profiles along the channel centerline of ion number density and ion mean velocity from the PIC simulation at point $1(\mathrm{t}=0.59 \mathrm{~ms})$.

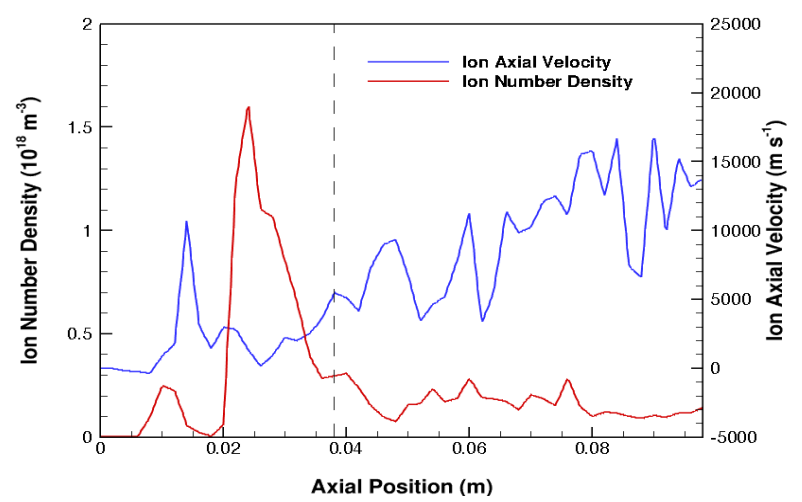

Figure 23. Profile along the channel centerline of ion number density and ion mean velocity from the PIC simulation at point $3(t=0.62 \mathrm{~ms})$.

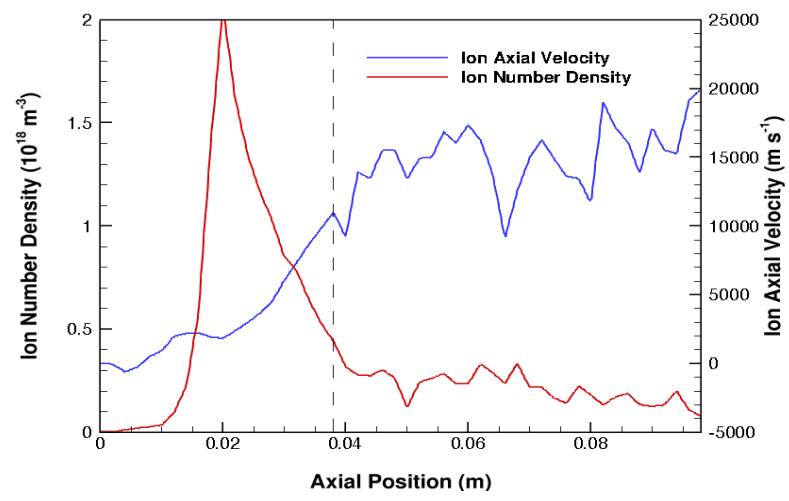

Figure 22. Profiles along the channel centerline of ion number density and ion mean velocity from the PIC simulation at point $2(t=0.60 \mathrm{~ms})$.

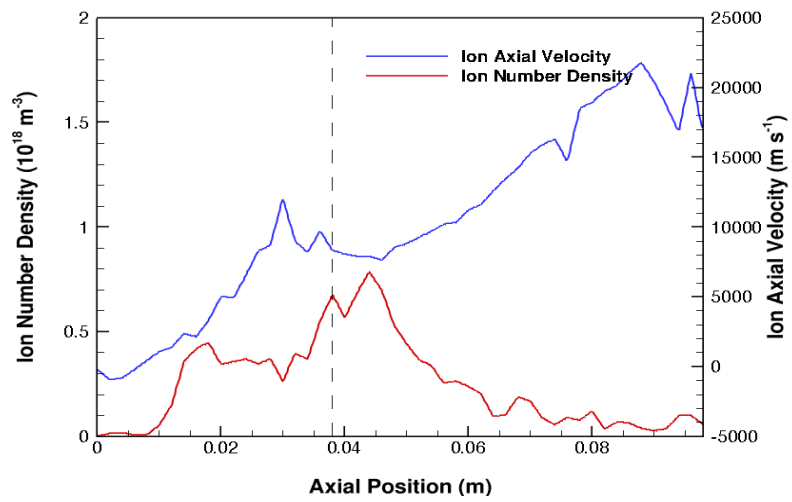

Figure 24. Profiles along the channel centerline of ion number density and ion mean velocity from the PIC simulation at point $4(t=0.63 \mathrm{~ms})$.

\section{Conclusions}

The present study describes initial attempts to verify a two-dimensional, axisymmetric hybrid-DK solver for the plasma physics in a Hall effect thruster channel and near field plume. Time-averaged plasma properties are slightly higher than the comparative hybrid-PIC simulation results. From the regularity of the discharge current, it is evident that DK results evolve smoothly in time, and plasma properties evolve continuously without any statistical noise. However, additional investigation must be performed with fewer constraints on quantities including neutral and ion number density. These constraints appear to affect the hybrid-DK plasma properties more significantly than those for the corresponding hybrid-PIC simulation, constraining the evolution of the plasma. The contributions of the ion and electron currents should be analyzed to determine their contributions to the high DK discharge current, and doubly charged ions should be added to the model. Additionally, the corresponding hybrid-PIC code's sensitivities to input values and numerical constraints should be further evaluated.

\section{Acknowledgments}

The authors gratefully acknowledge financial support provided by the Air Force Office of Scientific Research Grant No. F95550-09-1-0695.

\section{References}

${ }^{1}$ Fife, J., Hybrid-PIC Modeling and Electrostatic Probe Survey of Hall Thrusters, Ph.D. Thesis, Massachusetts Institute of Technology, 1999.

13 of 14

American Institute of Aeronautics and Astronautics 
${ }^{2}$ Hofer, R.R., Katz, I., Mikellides, I.G., Goebel, D.M., Jameson, K.K., Sullivan, R.M., and Johnson, L.K., "Efficacy of Electron Mobility Models in Hybrid-PIC Hall Thruster Simulations," AIAA Paper 2008-4924, Hartford, CT, July 2008.

${ }^{3}$ Hara, K., Development of Grid-Based Direct Kinetic Method and Hybrid Kinetic-Continuum Modeling of Hall Thruster Discharge Plasmas, Ph.D. Thesis, University of Michigan, 2015.

${ }^{4}$ Koo, J.W. and Boyd, I.D., "Modeling of Anomalous Electron Mobility in Hall Thrusters," Physics of Plasmas, Vol. 13, 2006, Article 033501.

${ }^{5}$ Hara, K., Boyd, I.D., and Kolobov, V.I., "One-dimensional hybrid direct kinetic simulation of the discharge plasma in a Hall thruster," Physics of Plasmas, Vol. 19, 2012, Article 113508.

${ }^{6}$ Puech, V. and Mizzi, S., "Collision Cross Sections and Transport Parameters in Neon and Xenon," Journal of Physics D: Applied Physics, Vol. 24, 1991, pp. 1974-1985.

${ }^{7}$ Koo, J.W., Hybrid PIC-MCC Computational Modeling of Hall Thrusters, Ph.D. Thesis, University of Michigan, 2005.

${ }^{8}$ Boeuf, J.P., and Garrigues, L., "Low Frequency Oscillations in a Stationary Plasma Thruster," Journal of Applied Physics, Vol. 84, No. 7, 1998, pp. 3541-3554.

${ }^{9}$ Ruyten, W.M., "Density-Conserving Shape Factors for Particle Simulations in Cylindrical and Spherical Coordinates", Journal of Computational Physics, Vol. 105, No. 2, 1993, pp. 224-232.

${ }^{10}$ Hofer, R.R., Katz, I., Mikellides, I.G., and Gamero-Castano, M., "Heavy Particle Velocity and Electron Mobility Modeling in Hybrid-PIC Hall Thruster Simulations,” AIAA Paper 2006-4658, Sacramento, CA, July 2006.

14 of 14

American Institute of Aeronautics and Astronautics 